\section{Models of pheochromocytoma: what's on the horizon?}
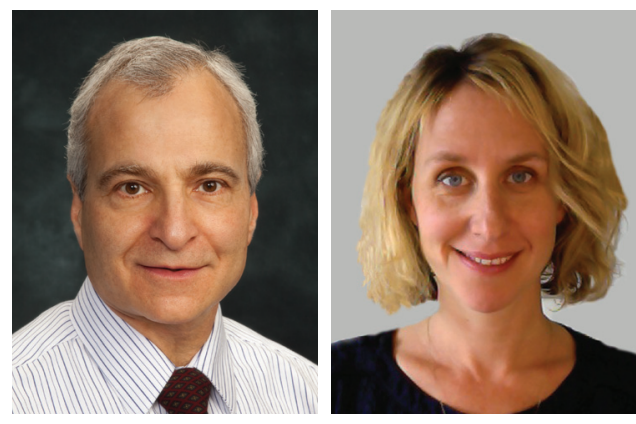

“There are currently no human

pheochromocytoma or

paraganglioma cell lines,

despite many efforts to

establish them and several

reports that were not

subsequently confirmed."

Arthur S Tischler ${ }^{*, 1}$ \& Judith Favier ${ }^{* *, 2}$

Pheochromocytomas (PCCs) and extraadrenal paragangliomas (PGLs) are the most hereditarily driven of all human tumors. Germline mutations of at least 15 genes are now known to confer susceptibility to these tumors in nearly half of affected patients [1]. Somatic mutations of the same genes and others account for a sizable percentage of the tumors that are sporadic [1,2]. Genotype-phenotype correlations associated with each driver mutation determine tumor location, biochemical function, multiplicity, metastatic potential and development of syndromically associated abnormalities. Although these correlations profoundly affect clinical decisions and patients' outcomes, development of effective treatments has unfortunately lagged behind the rapid advances in genetics of PCC/PGL. For patients in whom the tumors have metastasized, there is currently no cure.

The development of new treatment modalities will require new experimental models for both basic and translational research. Rodent models, human and animal cell lines, primary cell cultures and xenografts are complementary sources of information and could each play a role. The development of those models is particularly challenging given the tumors' diverse causes and manifestations. Nonetheless, progress has been made, in some cases by revisiting old models for new purposes. Rapidly evolving technologies also suggest that additional models might soon be available.

Potential animal models have existed since the 1960s in veterinary, toxicology, radiobiology and pathology literature. Historically, however, for the most part they were either regarded as curiosities or were utilized for research only tangentially related to clinical aspects of PCC or PGL as diseases. PCCs and, rarely, extra-adrenal PGLs, were reported to occur spontaneously in a variety of wild and domestic animal species, sometimes accompanied by hyperplasia or neoplasia in multiple endocrine glands in the same combinations seen in hereditary human endocrine neoplasia syndromes [3]. For most of the spontaneous models the genetic basis

\section{KEYWORDS}

- model - mouse - MPC cells

- paraganglioma - PC12 cells

- pheochromocytoma • rat

"Because of the increased sensitivity of dividing cells, the efficacy of many drugs tested thus far is likely to be overestimated by testing against [existing] cell lines..." 
"A priori, rats might be expected to be a more promising model than mice because of their innate proclivity to develop pheochromocytoma..." was not determined and relevance to human disease remains unknown. An exception is the MENX rat, which corresponds to MEN4 in humans and harbors a germline mutation in $C d k n 1 B[4,5]$.

Overall, spontaneous PCCs are unusually common in rats, particularly in aging males, but are rare in mice. It is therefore somewhat surprising that the tumors arise in a variety of genetically engineered mice, only some of which carry mutations known to be associated with human PCCs [3]. Further, some mice with relevant mutations do not display the phenotypes associated with those mutations in humans [6] or display incomplete or modified versions of those phenotypes [7]. A particular disappointment in that regard has been the absence of PCCs or PGLs in mice with succinate dehydrogenase gene mutations [6,8]. Because $S D H B$ mutations are the single most common cause of metastasizing PGLs in humans, SDHB models are sorely needed. Extra-adrenal PGLs are rare in both wild-type and genetically altered animals.

The cell lines currently most widely utilized as PCC models are PC12 [9], derived from an irradiated parabiotic rat, MPC 4/30/PRR (commonly abbreviated to simply MPC), from an irradiated heterozygous $N f 1$ knockout mouse [10] and MTT (mouse tumor tissue derived), a relatively aggressive derivative of MPC established from disseminated tumor developing after tail vein injection of MPC cells in a nude mouse [11]. Both PC12 and MPC proved to be genetically relevant to human PCC more than a decade after their development. PC12 cells were found early on to harbor a Max deletion [12], 17 years before $M A X$ was identified as a human PCC susceptibility gene [13]. Similarly, 12 years after the establishment of MPC cells it was discovered that NF1 is the most frequently mutated gene in sporadic human PCC [14]. There are currently no human PCC or PGL cell lines, despite many efforts to establish them and several reports that were not subsequently confirmed. One existing human line, obtained by transducing primary cultures of sporadic PCC cells with hTERT, so far appears to be at best minimally functional and was reported as a progenitor cell line [15].

Where do we stand today and where are we headed? As complementary models, MPC and MTT cells are already used in preclinical studies to test candidate drugs for treating metastatic PCC. Their use for that purpose is likely to increase. PC12 cells, for years studied mainly in the domain of neuroscience, are also increasingly used for drug testing. However, a caveat is that all three cell lines proliferate rapidly compared with human PCCs, which typically grow slowly in vivo and not at all in culture [16]. Because of the increased sensitivity of dividing cells, the efficacy of many drugs tested thus far is likely to be overestimated by testing against these cell lines, as illustrated by a recent study comparing cytotoxicity of topoisomerase 1 inhibitors against MPC, MTT and primary human PCC/ PGL cell cultures [16]. These rodent cell lines and newer ones will increasingly be used to search for more tumor-specific targeted therapies. At a more basic level, PC12 and MPC cells are being utilized to dissect signaling pathways responsible for specific aspects of tumor phenotype. In elegant studies Qin et al. have shown that introduction of Max to PC12 cells, which are noradrenergic, increased PNMT expression and slowed proliferation while introduction of Hif $2 a$ to MPC cells, in which that gene is lacking, caused impaired PNMT expression and increased proliferation [17].

Although PCC/PGL cell lines are not yet available to represent the entire gamut of mutations, lines of other cell types harboring those mutations will help to fill the gap. Dahia et al. have studied fibroblasts from a new Tmem127 knockout mouse and TMEM127-mutated human renal cell carcinoma to show how loss of TMEM127 causes accumulation of active mTOR in lysosomes, suggesting possible therapeutic strategies targeting endosomal/lysosomal pathways [18]. Favier et al. have utilized nonfunctional immortalized $S d h b^{-1-}$ cells from mouse adrenal medulla to show that increased succinate accumulation in these cells inhibits DNA and histone demethylation, leading to a hypermethylator phenotype comparable to that seen in SDHB mutated human PGL and supporting therapeutic use of demethylating agents [19]. These cells have a slow proliferation rate associated with increased migratory and invasion abilities and have been established in a xenograft model in nude mice [FAVIer Laboratory, Unpublished Data]. A revolutionary technological advance likely to speed the development of new models is the advent of the genome-editing techniques TALEN and CRISPR, which permit precise insertion or deletion of genes or portions of genes at specific sites [20]. These new techniques can both speed the development and lower the cost of animal models by directly modifying the 
genome in an ovum, in contrast to conventional methods starting with embryonic stem cells. In cell cultures they permit complete knockout of genes, in contrast to knockdown obtainable with siRNA, and probably with fewer off-target effects [20]. In one project already underway, heterozygous $S d h b^{+/-}$rats have been developed using the TALEN technique [Powers JF, Tischler as, Unpublished Data]. A priori, rats might be expected to be a more promising model than mice because of their innate proclivity to develop PCC and, occasionally, PGL. Further, the incidence of PCC in rats is increased by diet, hormones, drugs and other chemicals [3]. If these animals do develop $S d h b$-associated tumors they will therefore serve as both a source of needed cell lines and a novel model for studying tumor induction and progression at its earliest stages.

Despite many advances, it is inescapable that human PCC or PGL cell lines would be preferable to rodent lines or lines of phenotypically dissimilar cells. The absence of these cell lines presents both a source of frustration and a conundrum. Hundreds of attempts using many techniques and culture media since at least the early 1970s have demonstrated that human PCC or PGL cells do not proliferate in culture and do not take in mouse xenografts. The conundrum is why the tumor cells, even from tumors that grew rapidly in vivo, immediately cease to proliferate in culture but can often survive for a year or longer while maintaining tyrosine hydroxylase expression and other differentiated traits. Immunocytochemical staining for bromodeoxyuridine incorporation and tyrosine hydroxylase expression demonstrates that cessation of DNA synthesis and tumor cell proliferation occurs less than one day in vitro, and is therefore likely attributable to causes other than cellular senescence. Importantly, neither hypoxia nor patient-derived serum is mitogenic. Current approaches to deciphering the conundrum include transduction of primary cultures with lentivirus libraries designed either to screen multiple kinases for ability to activate proliferation or to screen multiple siRNAs for ability to knock down inhibitors of proliferation. Systematic efforts to identify metabolomic and transcriptional differences between tumor tissue and cultured tumor cells are also in their early phases. Preliminary attempts have also been made in some laboratories to generate cell lines and animal models starting with induced pluripotent stem cells from patients with hereditary PCC or PGL. It remains to be seen whether any of these novel approaches will bear fruit.

The international PCC/PGL research consortium PRESSOR encourages and attempts to foster collaboration on development of new experimental models (for more information, please visit the PRESSOR Tumor Models Working Group webpage [21]).

\section{Financial \& competing interests disclosure}

A Tischler and J Favier are co-Chairs of the PRESSOR Tumor Models Working Group. The authors have no other relevant affiliations or financial involvement with any organization or entity with a financial interest in or financial conflict with the subject matter or materials discussed in the manuscript apart from those disclosed.

No writing assistance was utilized in the production of this manuscript.

\section{References}

1 Favier J, Amar L, Gimenez-Roqueplo AP. Paraganglioma and phaeochromocytoma: from genetics to personalized medicine. Nat. Rev. Endocrinol. 11(2), 101-111 (2015).

2 Dahia PL. Pheochromocytoma and paraganglioma pathogenesis: learning from genetic heterogeneity. Nat. Rev. Cancer 14(2), 108-119 (2014).

3 Tischler AS, Powers JF, Alroy J. Animal models of pheochromocytoma. Histol. Histopathol. 19(3), 883-895 (2004).

4 Pellegata NS, Quintanilla-Martinez L, Siggelkow $\mathrm{H}$ et al. Germ-line mutations in p27Kip1 cause a multiple endocrine neoplasia syndrome in rats and humans. Proc. Natl Acad. Sci. USA 103(42), 15558-15563 (2006).
5 Molatore S, Liyanarachchi S, Irmler M et al. Pheochromocytoma in rats with multiple endocrine neoplasia (MENX) shares gene expression patterns with human pheochromocytoma. Proc. Natl Acad. Sci. USA 107(43), 18493-18498 (2010).

6 Bayley JP, Van Minderhout I, Hogendoorn PC et al. Sdhd and SDHD/H19 knockout mice do not develop paraganglioma or pheochromocytoma. PLoS ONE 4(11), e7987 (2009).

7 Smith-Hicks CL, Sizer KC, Powers JF, Tischler AS, Costantini F. C-cell hyperplasia, pheochromocytoma and sympathoadrenal malformation in a mouse model of multiple endocrine neoplasia type 2B. EMBO J. 19(4), 612-622 (2000).
8 Maher LJ III, Smith EH, Rueter EM et al. Mouse models of human familial paraganglioma. In: Mouse Models of Human Familial Paraganglioma, Pheochromocytoma - A New View of the Old Problem. Martin, JF (Ed.). InTech (2011).

9 Greene LA, Tischler AS. Establishment of a noradrenergic clonal line of rat adrenal pheochromocytoma cells which respond to nerve growth factor. Proc. Natl Acad. Sci. USA 73(7), 2424-2428 (1976).

10 Powers JF, Evinger MJ, Tsokas P et al. Pheochromocytoma cell lines from heterozygous neurofibromatosis knockout mice. Cell Tissue Res. 302(3), 309-320 (2000).

11 Martiniova L, Lai EW, Elkahloun AG et al. Characterization of an animal model of 
EDITORIAL Tischler \& Favier

aggressive metastatic pheochromocytoma linked to a specific gene signature. Clin. Exp. Metastasis 26(3), 239-250 (2009).

12 Hopewell R, Ziff EB. The nerve growth factor-responsive PC12 cell line does not express the Myc dimerization partner Max. Mol. Cell. Biol. 15(7), 3470-3478 (1995).

13 Burnichon N, Cascon A, Schiavi F et al. Max mutations cause hereditary and sporadic pheochromocytoma and paraganglioma. Clin. Cancer Res. 18(10), 2828-2837 (2012).

14 Burnichon N, Buffet A, Parfait B et al. Somatic NF1 inactivation is a frequent event in sporadic pheochromocytoma. Hum. Mol. Genet. 21(26), 5397-5405 (2012).

15 Ghayee HK, Bhagwandin VJ, Stastny V et al. Progenitor cell line (hPheol) derived from a human pheochromocytoma tumor. PLoS ONE 8(6), e65624 (2013).

16 Powers JF, Korgaonkar PG, Fliedner S, Giubellino A, Sahagian KP, Tischler AS. Cytocidal activities of topoisomerase 1 inhibitors and 5-azacytidine against pheochromocytoma/paraganglioma cells in primary human tumor cultures and mouse cell lines. PLoS ONE 9(2), e87807 (2014).

17 Qin N, De Cubas AA, Garcia-Martin R et al. Opposing effects of HIFl $\alpha$ and $H I F 2 \alpha$ on chromaffin cell phenotypic features and tumor cell proliferation: insights from MYC-associated factor X. Int. J. Cancer 135(9), 2054-2064 (2014).
18 Qin Y, Deng Y, Ricketts CJ et al. The tumor susceptibility gene TMEM127 is mutated in renal cell carcinomas and modulates endolysosomal function. Hum. Mol. Genet. 23(9), 2428-2439 (2014).

19 Letouze E, Martinelli C, Loriot C et al. SDH mutations establish a hypermethylator phenotype in paraganglioma. Cancer Cell 23(6), 739-752 (2013).

20 Wei C, Liu J, Yu Z, Zhang B, Gao G, Jiao R. TALEN or Cas9 - rapid, efficient and specific choices for genome modifications. J. Genet. Genomics 40 (6), 281-289 (2013).

21 PRESSOR.

www.pressor.org/ 